Preprint typeset in JHEP style - HYPER VERSION

\title{
Group Averaging of massless scalar fields in $1+1$ de Sitter
}

\author{
Donald Marolf and lan A Morrison \\ Department of Physics, University of California at Santa Barbara, Santa Barbara, CA \\ 93106, USA \\ E-mail: marolf@physics.ucsb.edu, ian_morrison@physics.ucsb.edu
}

\begin{abstract}
Perturbative gravity in global de Sitter space is subject to so-called linearization stability constraints: If they are to couple consistently to the gravitational field, quantum states must be invariant under the de Sitter isometries. While standard Fock spaces contain no de Sitter-invariant states apart from (possibly) the vacuum, a full Hilbert space of de Sitter-invariant quantum states can be constructed via group averaging techiniques. We re-examine the simple toy model of de Sitter group averaging given by the free $1+1$ scalar field, expanding on an earlier analysis by Higuchi. Our purpose is twofold: to include the scalar zero-mode, and to explicitly count the number of de Sitter-invariant states as a function of an appropriately defined energy.
\end{abstract}

KEYWORDS: de Sitter, curved space quantum field theory, linearization stability, group averaging. 


\section{Contents}

1. Introduction 1

2. Free scalar field in $1+1$ de Sitter 3

2.1 Operators and States 3

2.2 The de Sitter group

3. Group Averaging and the physical Hilbert space 6

4. Physical Entropy 8

5. Discussion 10

\section{Introduction}

Understanding quantum gravity in de Sitter space remains an important problem. A major motivation is the relevance of de Sitter to cosmology: measurements of the CMB [四] are consistent with a period of inflation in which the universe underwent a de Sitter-like phase of rapid expansion, and observations of type Ia supernovae suggest [2] that our universe may have a small positive cosmological constant and may approach de Sitter space in the far future. Thus any theory of quantum gravity should include a description of de Sitter space, at least in some approximate form. Unfortunately, the study of de Sitter quantum gravity has been fraught with conceptual difficulties (see, e.g., 3, 4, 5]). In this paper we examine one particular hurdle that arises in perturbative gravity about a de Sitter background.

To summarize this hurdle, recall that field theories on spacetimes with Killing symmetries have conserved charges. We wish to regard such a theory (together with linearized gravitational waves) as the zero-order perturbative approximation to a theory of matter plus gravity. This context is particularly interesting when the background also has compact Cauchy surfaces. Then the gravitational equivalent of Gauss' law implies that the above charges must vanish in order for a solution to this zero-order theory to consistently couple to dynamical gravity [6, 7, 8, 9, 10, 11, 12]. Since these constraints are not encoded in the linearized field equations, they are known as linearization-stability constraints.

In de Sitter space, the linearization-stability constraints require linearized quantum states to be invariant under the de Sitter group $S O_{0}(D, 1)$ where $D$ is the spacetime dimension [13, 14, 15]. Because the de Sitter group is non-compact, the standard Fock space contains no de Sitter-invariant states except for a possible vacuum [14]. This meager set of states is clearly insufficient to reproduce the rich physics of the corresponding classical 
theory. Fortunately, however, one may use the standard Fock space (which we call the 'auxiliary' Hilbert space $\mathcal{H}_{\text {aux }}$ ) to build a new 'physical' Hilbert space $\mathcal{H}_{\text {phys }}$ of de Sitterinvariant states via group averaging $[14]^{1}$. This technique considers linear superpositions of auxiliary states $\llbracket \psi\rangle$ of the form

$$
\left.|\Psi\rangle:=\int_{g \in G} d g U(g) \llbracket \psi\right\rangle,
$$

where $G$ is the de Sitter group, $d g$ is the unique (unimodular, left- and right-invariant) Haar measure of $G$, and $U(g)$ gives the unitary representation of $G$ on $\mathcal{H}_{\text {aux }}$. Such superpositions are formally invariant under the de Sitter group. For compact groups the analogue of (1.1) converges and gives the projection of $\llbracket \psi\rangle$ onto the trivial representation. However, since our $G$ is non-compact, the state (1.1) is not normalizable in $\mathcal{H}_{\text {aux }}$. Nevertheless it can be understood (see e.g. [18]) as a "generalized state" in a sense similar to that used for non-normalizable eigenstates of operators with continuous spectrum (e.g., plane waves in infinite space).

More concretely, one defines a new inner product on the group-averaged states (1.1):

$$
\left\langle\Psi_{1} \mid \Psi_{2}\right\rangle:=\left\langle\psi_{1} \rrbracket \cdot \mid \Psi_{2}\right\rangle=\int_{g \in G} d g\left\langle\psi_{1} \rrbracket U(g) \llbracket \psi_{2}\right\rangle .
$$

The linear superposition (1.1) is meaningful when this "group-averaging inner product" converges.

When the sense of the convergence is sufficiently strong, a theorem of [19] states that the group-averaging inner product is the unique inner product consistent with the $\star$ algebra of bounded gauge-invariant observables in $\mathcal{H}_{\text {aux }}$. More formal discussions of group averaging can be found in [20, 21]. Other studies of de Sitter group averaging include $22,23$.

The purpose of this paper is to analyze group averaging for a massless scalar field in $1+1$ de Sitter, completing the analysis begun by Higuchi [14]. Higuchi was primarily concerned with de Sitter group averaging for $3+1$ gravitons and used the $1+1$ massless scalar as a toy model. For simplicity, he omitted the scalar zero mode (which has no analogue for gravitons). However, the physical massless scalar has a zero mode that should be included in a more complete analysis. We do so below. We also compute the number of de Sitter-invariant states as a function of energy flux through the de Sitter neck. For energies much greater than the de Sitter scale, a straightforward calculation shows that this entropy agrees with that of the naive auxiliary Hilbert space. This provides an explicit check of the argument presented in [22] that such a result should hold for generic field theories.

This paper is organized as follows. Section 2 briefly reviews the quantization of the massless scalar in de Sitter. Section 3 then follows Higuchi in using group averaging to construct an orthonormal basis of physical states from special auxiliary "seed states." The physical entropy is computed in 1 and section 5 presents some final discussion.

\footnotetext{
${ }^{1}$ See [16, 17] for independent introductions of similar techniques in related contexts.
} 


\section{Free scalar field in $1+1$ de Sitter}

We begin with a brief overview of massless scalar fields in $1+1$ de Sitter [24, 25, 26]. It is useful to adopt conventions of conformal field theory [27, 28], and to write the $1+1$ de Sitter metric in the form

$$
d s^{2}=\frac{\ell^{2}}{\cos ^{2} \tau}\left(-d \tau^{2}+d \theta^{2}\right)
$$

which is just a conformal factor times the metric on the cylinder. Here the conformal time $\tau$ has range $-\pi / 2<\tau<\pi / 2, \theta$ periodic $\theta \cong \theta+2 \pi$, and $\ell$ is the de Sitter length scale. We adopt lightcone coordinates $x^{ \pm}=\tau \pm \theta$.

\subsection{Operators and States}

The action of a free scalar field is

$$
S=-\frac{1}{2} \int d^{2} x \sqrt{-g} g^{a b} \nabla_{a} \phi \nabla_{b} \phi=\int d^{2} x \partial_{+} \phi \partial_{-} \phi,
$$

where $g_{a b}$ is the de Sitter metric and $\nabla_{a}$ the covariant derivative associated with $g_{a b}$. In the second equality we note that the conformal factor from (2.1) cancels out of the action, making the theory conformally invariant. The equation of motion for $\phi$ is thus $\partial_{+} \partial_{-} \phi(x)=0$, and the solutions are familiar left- and right-moving modes

$$
\partial_{+} \phi\left(x^{+}\right)=\frac{1}{2 \sqrt{\pi}} \sum_{m} \alpha_{m} \exp \left[-i m x^{+}\right], \quad \partial_{-} \phi\left(x^{-}\right)=\frac{1}{2 \sqrt{\pi}} \sum_{m} \widetilde{\alpha}_{m} \exp \left[-i m x^{-}\right] .
$$

Upon integrating one finds

$$
\begin{aligned}
\phi(x)= & \frac{\phi_{0}}{4 \pi}+\alpha_{0} x^{+}+\widetilde{\alpha}_{0} x^{-}+\frac{i}{2 \sqrt{\pi}} \sum_{m \neq 0}\left[\frac{\alpha_{m}}{m} e^{-i m x^{+}}+\frac{\widetilde{\alpha}_{m}}{m} e^{-i m x^{-}}\right] \\
= & \frac{\phi_{0}}{4 \pi}+\left(\alpha_{0}+\widetilde{\alpha}_{0}\right) \tau+\left(\alpha_{0}-\widetilde{\alpha}_{0}\right) \theta \\
& +\frac{i}{2 \sqrt{\pi}} \sum_{m \neq 0} \frac{1}{m} e^{-i m \tau}\left[\alpha_{m} e^{-i m \theta}+\widetilde{\alpha}_{m} e^{+i m \theta}\right] .
\end{aligned}
$$

We identify the term linear in $\tau$ as the momentum $p \propto\left(\alpha_{0}+\widetilde{\alpha}_{0}\right)$. The fact that $\phi(x)$ must be single-valued places further constraints on the mode expansion, depending on the target space of $\phi(x)$. We consider two cases:

i) The target space of $\phi(x)$ is the real line. Single-valuedness of $\phi(x)$ requires $\phi(\tau, \theta+$ $2 \pi)=\phi(\tau, \theta)$; thus $\alpha_{0}=\widetilde{\alpha}_{0}$ and the term linear in $\theta$ in (2.4) vanishes.

ii) The target space of $\phi(x)$ is the circle $S^{1}$ with radius $R$. Single-valuedness requires $\phi(\tau, \theta+2 \pi)=\phi(\tau, \theta)+2 \pi R w$, where $w \in \mathbb{Z}$ is the winding number of the field. From (2.4) we see that $R w$ is given by $R w=\left(\alpha_{0}-\widetilde{\alpha}_{0}\right)$. Furthermore, because $\phi(x)$ is periodic, $p$ is quantized: $p=k / R, k \in \mathbb{Z}$. Periodic scalars in de Sitter have previously been considered in, e.g., [29]. 
For the remainder of this section we will keep $p$ explicit so that our expressions apply to either case; later we will specialize to case (ii) and write expressions in terms of $k$. Our mode expansion is now

$$
\phi(x)=\frac{\phi_{0}}{4 \pi}+2 p \tau+R w \theta+\frac{i}{2 \sqrt{\pi}} \sum_{m \neq 0} \frac{1}{m} e^{-i m \tau}\left[\alpha_{m} e^{-i m \theta}+\widetilde{\alpha}_{m} e^{i m \theta}\right] .
$$

We now quantize our scalar field using canonical techniques, the end result of which is the auxiliary Hilbert space $\mathcal{H}_{\text {aux }}$. The quantities $\phi_{0}, p, w, \alpha_{m}$, and $\widetilde{\alpha}_{m}$ are promoted to operators. Imposing the canonical commutation relation $\left[\phi\left(\tau, \theta_{1}\right), \phi\left(\tau, \theta_{2}\right)\right]=i \delta\left(\theta_{1}-\theta_{2}\right)$ we find

$$
\left[\phi_{0}, p\right]=i, \quad\left[\alpha_{m}, \alpha_{n}\right]=\left[\widetilde{\alpha}_{m}, \widetilde{\alpha}_{n}\right]=m \delta_{m,-n},
$$

with all other commutators vanishing. In the usual fashion, $\alpha_{m}$ and $\widetilde{\alpha}_{m}$ are interpreted as left- and right- moving creation operators $(m<0)$ and annihilation operators $(m>0)$. It will be useful to use the Virasoro generators $L_{0}, L_{ \pm 1}$ [27, 28]

$$
L_{m}=\frac{1}{2} \sum_{n=-\infty}^{\infty}: \alpha_{m-n} \alpha_{n}:
$$

which obey the algebra

$$
\left[L_{ \pm 1}, L_{0}\right]= \pm L_{ \pm 1}, \quad\left[L_{1}, L_{-1}\right]=2 L_{0}
$$

and likewise for $\widetilde{L}_{0}, \widetilde{L}_{ \pm 1}$.

We can define a vacuum state $\llbracket 0\rangle$ as the state for which

$$
\left.\left.\alpha_{m} \rrbracket 0\right\rangle=\widetilde{\alpha}_{m} \rrbracket 0\right\rangle=0 \quad \forall m>0 .
$$

Such a vacuum state is not in general annihilated by $\alpha_{0}$ or $\widetilde{\alpha}_{0}$. Instead, there is a twoparameter family of vacua distinguished by their eigenvalues of $\alpha_{0}$ and $\widetilde{\alpha}_{0}$, i.e. the momentum and winding of each vacuum. It is equivalent to label independent vacua by their eigenvalues $h$ and $\widetilde{h}$ of the Virasoro generators $L_{0}$ and $\widetilde{L}_{0}$ :

$$
h=\frac{1}{2}\left(p+\frac{R w}{2}\right)^{2}, \quad \widetilde{h}=\frac{1}{2}\left(p-\frac{R w}{2}\right)^{2} ;
$$

we therefore denote a vacuum by $\llbracket 0 ; h, \widetilde{h}\rangle$. We shall see shortly that the only de Sitterinvariant vacuum is the $p=w=0$ vacuum $\llbracket 0 ; 0,0\rangle$. Excited states are created by acting on a vacuum with creation operators $\alpha_{m}\left(\widetilde{\alpha}_{m}\right)$ for $m<0$, and will be labeled using the somewhat degenerate notation $\llbracket n, \widetilde{n} ; h, \widetilde{h}\rangle$, where $n, \tilde{n}$ are the eigenvalues of $L_{0}-h, \widetilde{L}_{0}-\tilde{h}$ and we refer to $N:=n+\widetilde{n}$ as the level of a state. Each creation operator $\alpha_{m}\left(\widetilde{\alpha}_{m}\right)$ increases the eigenvalue of $L_{0}\left(\widetilde{L}_{0}\right)$, and thus the level, by $m$.

Let us also introduce the operator

$$
H=L_{0}+\widetilde{L}_{0}
$$


which generates translations in $\tau$; i.e., it is the Hamiltonian for the conformally rescaled problem on the cylinder $S^{1} \times \mathbb{R}$, up to a constant offset associated with the Casimir energy. Since de Sitter space does not have a global future-directed timelike Killing field, $H$ is not naturally thought of as a de Sitter Hamiltonian. However, it does agree with the flux of de Sitter stress-energy through the sphere at $\tau=0$ (again up to a constant offset). In this latter form, this operator was an important ingredient in the analysis of [22]. We shall thus refer to $H$ as an "energy." This operator acts on a state $\llbracket n, \widetilde{n} ; h, \widetilde{h}\rangle$ as

$$
\begin{aligned}
H \llbracket n, \widetilde{n} ; h, \widetilde{h}\rangle & =(h+\widetilde{h}+n+\widetilde{n}) \llbracket n, \widetilde{n} ; h, \widetilde{h}\rangle \\
& \left.=\left(p^{2}+\frac{R^{2} w^{2}}{4}+n+\widetilde{n}\right) \llbracket n, \widetilde{n} ; h, \widetilde{h}\right\rangle,
\end{aligned}
$$

and so the energy of such a state is $E:=h+n+\widetilde{h}+\widetilde{n}$.

\subsection{The de Sitter group}

Let us quickly review the symmetries of $1+1$ de Sitter spacetime. This space has three independent Killing vector fields which we may take to be

$\partial_{\theta}=\frac{1}{2}\left(\partial_{+}-\partial_{-}\right), \quad \xi_{1}^{a} \partial_{a}=\frac{1}{2}\left(\cos \left(x^{+}\right) \partial_{+}+\cos \left(x^{-}\right) \partial_{-}\right), \quad \xi_{2}^{a} \partial_{a}=\frac{1}{2}\left(\sin \left(x^{+}\right) \partial_{+}+\cos \left(x^{-}\right) \partial_{-}\right)$.

Such isometries can be understood by embedding $1+1$ de Sitter in $2+1$ Minkowski space: there $\partial_{\theta}$ generates rotations preserving the Cartesian coordinate $X^{0}$, while $\xi_{1}^{a}$ and $\xi_{2}^{a}$ generate boosts along the Cartesian spatial directions. The Killing fields act on $\mathcal{H}_{\text {aux }}$ via operators $J, B_{1}$, and $B_{2}$ which satisfy the $S O_{0}(2,1)$ algebra

$$
\left[B_{1}, B_{2}\right]=i J, \quad\left[B_{1}, J\right]=i B_{2}, \quad\left[B_{2}, J\right]=-i B_{1} .
$$

On the scalar field $\phi(x)$, their action is

$$
\left[B_{1}, \phi(x)\right]=i £_{\xi_{1}} \phi(x)=i \xi_{1}^{a} \partial_{a} \phi(x)
$$

and likewise for $J$ and $B_{2}$. One may express the $S O_{0}(2,1)$ generators in terms of Virasoro generators via

$$
\begin{aligned}
J & =L_{0}-\widetilde{L}_{0}, \\
B_{1} & =\frac{1}{2}\left(L_{1}+L_{-1}+\widetilde{L}_{1}+\widetilde{L}_{-1}\right), \\
B_{2} & =-\frac{i}{2}\left(L_{1}-L_{-1}-\widetilde{L}_{1}+\widetilde{L}_{-1}\right) .
\end{aligned}
$$

We see that the de Sitter group is a diagonal subgroup of the $S L(2, C) \times S L(2, C)$ generated by $L_{0}, L_{ \pm 1}, \widetilde{L}_{0}, \widetilde{L}_{ \pm 1}$.

Constructing de Sitter-invariant states is non-trivial, as can be seen from the expressions of the generators (2.16)-(2.18). Because the boost generators contain both raising and lowering Virasoro generators, it is difficult to construct a non-trivial state that is boost 
invariant. Indeed, it is easy to show that the only de Sitter-invariant state in our basis is the $p=w=0$ vacuum $\llbracket 0 ; 0,0\rangle$ (see also [30]). Furthermore, it can be shown that there exist no linear combinations of our basis states that are both de Sitter-invariant and normalizable [13]. Thus $\llbracket 0 ; 0,0\rangle$ is the only de Sitter-invariant state in the auxiliary Hilbert space $\mathcal{H}_{\text {aux }}$.

\section{Group Averaging and the physical Hilbert space}

We now construct de Sitter invariant states via group averaging. We study the resulting physical Hilbert space $\mathcal{H}_{\text {phys }}$ and provide an orthonormal basis. We follow closely in the steps of [14] and, in particular, define the space $\left.\mathcal{H}_{\text {seed }}=\{\rrbracket \psi\rangle_{\text {seed }}\right\}$ of "Higuchi seed states" which are:

i) $S O(2)$-invariant, i.e.

$$
J \rrbracket \psi\rangle_{\text {seed }}=0,
$$

ii) annihilated by the lowering operators $L_{1}$ and $\widetilde{L}_{1}$,

$$
\left.\left.L_{1} \rrbracket \psi\right\rangle_{\text {seed }}=\widetilde{L}_{1} \rrbracket \psi\right\rangle_{\text {seed }}=0,
$$

iii) in the subspace corresponding to eigenvalues $E>1$ of $H$ (recall 2.12). We note that $H$ preserves the conditions (3.1), (3.2) and so can be diagonalized in $\mathcal{H}_{\text {seed }}$.

Furthermore, we will confine attention to a basis of such states which are eigenstates of $E$ with inner products

$$
{ }_{\text {seed }}\left\langle\psi_{1} \rrbracket \psi_{2}\right\rangle_{\text {seed }}=\frac{(E-1)}{2} \delta_{\psi_{1}, \psi_{2}} .
$$

Here $\delta_{\psi_{1}, \psi_{2}}$ denotes the complete set of Kronecker deltas needed to specify that $\left.\llbracket \psi_{1}\right\rangle_{\text {seed }}$ and $\left.\llbracket \psi_{2}\right\rangle_{\text {seed }}$ represent the same state in our basis .

The group averaging of such states is easy to control. Criterion (ii.) has the effect that

$$
\left.\left.B_{1} \rrbracket \psi\right\rangle_{\text {seed }}=\left(L_{-1}+\widetilde{L}_{-1}\right) \rrbracket \psi\right\rangle_{\text {seed }}, \quad \text { seed }\left\langle\psi \rrbracket B_{1}={ }_{\text {seed }}\left\langle\psi \rrbracket\left(L_{1}+\widetilde{L}_{1}\right) .\right.\right.
$$

As a result seed $\left\langle\psi_{1} \llbracket B_{1} \rrbracket \psi_{2}\right\rangle_{\text {seed }}=0$ for all seed states. Recalling the commutation relations

$$
\left[L_{ \pm 1}, L_{0}\right]= \pm L_{ \pm 1}, \quad\left[L_{1}, L_{-1}\right]=2 L_{0}
$$

we may compute seed $\left\langle\psi_{1} \rrbracket\left(B_{1}\right)^{2} \rrbracket \psi_{2}\right\rangle_{\text {seed }}$ by commuting creation operators to the left (annihilation operators to the right), with the result seed $\left\langle\psi_{1} \rrbracket\left(B_{1}\right)^{2} \rrbracket \psi_{2}\right\rangle_{\text {seed }}={ }_{\text {seed }}\left\langle\psi_{1} \rrbracket\left(L_{0}+\right.\right.$ $\left.\left.\widetilde{L}_{0}\right) \rrbracket \psi_{1}\right\rangle_{\text {seed }}=E_{\text {seed }}\left\langle\psi_{1} \rrbracket \psi_{2}\right\rangle_{\text {seed }}$, where $E$ is the energy of either state. Continuing in this manner, one can readily see that

$$
\begin{aligned}
& \text { seed }\left\langle\psi_{1} \rrbracket\left(B_{1}\right)^{m} \rrbracket \psi_{2}\right\rangle_{\text {seed }}=0 \quad m \text { odd, } \\
& \text { seed }\left\langle\psi_{1} \rrbracket\left(B_{1}\right)^{m} \rrbracket \psi_{2}\right\rangle_{\text {seed }}=f(m, E)_{\text {seed }}\left\langle\psi_{1} \rrbracket \psi_{2}\right\rangle_{\text {seed }} \quad m \text { even, }
\end{aligned}
$$


where $f(m, E)$ is a function of $m$ and $E$. In particular, [14] showed that

$$
{ }_{\text {seed }}\left\langle\psi_{1} \rrbracket e^{i \lambda B_{1}} \rrbracket \psi_{2}\right\rangle_{\text {seed }}=\left(\cosh \frac{\lambda}{2}\right)^{-2 E} \text { seed }\left\langle\psi_{1} \rrbracket \psi_{2}\right\rangle_{\text {seed }}
$$

if the zero-mode is ignored, i.e. for the Fock space over the $p=w=0$ vacuum. However, since dependence on $p, w$ enters only through $E$, we see that (3.8) holds in general. Working in terms of the the energy flux $E$ turns out to make the inclusion of several effects of the zero mode quite straightforward.

We wish to compute the group averaging inner product of two seed states. We begin by specializing expression (1.2) to the case of $S O_{0}(2,1)$. Using the Cartan decomposition of $S O_{0}(2,1)$ we can write any group element as a product of two $S O(2)$ rotations and a boost [31, 32]:

$$
U(g)=e^{i \alpha J} e^{i \lambda B_{1}} e^{i \gamma J} .
$$

Here $e^{i \alpha J}$ is the $S O(2)$ rotation through angle $\alpha(0 \leq \alpha \leq 2 \pi)$ and $e^{i \lambda B_{1}}$ is the boost along $\xi_{1}^{a}$ with rapidity $\lambda(0 \leq \lambda \leq \infty)$. In a similar fashion, the Haar measure can be decomposed as

$$
d g=\frac{1}{4 \pi^{2}} d \alpha d \gamma d \lambda \sinh \lambda,
$$

where $\frac{1}{2 \pi} d \alpha$ and $\frac{1}{2 \pi} d \gamma$ are both Haar measures on $S O(2)$. The group averaged inner product is then

$$
\begin{aligned}
\left\langle\Psi_{1} \mid \Psi_{2}\right\rangle & =\frac{1}{4 \pi^{2}} \int d \alpha d \gamma d \lambda \sinh \lambda\left\langle\psi_{1} \rrbracket e^{i \alpha J} e^{i \lambda B_{1}} e^{i \gamma J} \rrbracket \psi_{2}\right\rangle \\
& =\int_{0}^{\infty} d \lambda \sinh \lambda\left\langle\psi_{1} \llbracket \mathcal{P}_{0} e^{i \lambda B_{1}} \mathcal{P}_{0} \rrbracket \psi_{2}\right\rangle
\end{aligned}
$$

In the second line we have identified the projector $\mathcal{P}_{0}$ onto $S O(2)$-invariant states

$$
\mathcal{P}_{0}=\frac{1}{2 \pi} \int_{0}^{2 \pi} d \alpha e^{i \alpha J}
$$

Now consider the inner product of two physical states built from seed states $\left|\Psi_{1,2}\right\rangle:=$ $\left.\int d g U(g) \rrbracket \psi_{1,2}\right\rangle_{\text {seed }}$. From (3.11) we have

$$
\begin{aligned}
\left\langle\Psi_{1} \mid \Psi_{2}\right\rangle & =\int_{0}^{\infty} d \lambda \sinh \lambda_{\text {seed }}\left\langle\psi_{1} \llbracket \mathcal{P}_{0} e^{i \lambda B_{1}} \mathcal{P}_{0} \rrbracket \psi_{2}\right\rangle_{\text {seed }} \\
& =\int_{0}^{\infty} d \lambda \sinh \lambda_{\text {seed }}\left\langle\psi_{1} \rrbracket e^{i \lambda B_{1}} \rrbracket \psi_{2}\right\rangle_{\text {seed }} \\
& =\operatorname{seed}\left\langle\psi_{1} \rrbracket \psi_{2}\right\rangle_{\text {seed }} \int_{0}^{\infty} d \lambda \sinh \lambda\left(\cosh \frac{\lambda}{2}\right)^{-2 E} \\
& ={ }_{\text {seed }}\left\langle\psi_{1} \rrbracket \psi_{2}\right\rangle_{\text {seed }} \frac{2}{(E-1)} \\
& =\delta_{\psi_{1}, \psi_{2}} .
\end{aligned}
$$

In the second line we used the fact that seed states are $S O(2)$-invariant; in the third line we used (3.8). Evaluating the integral and inserting our normalization (3.3) leads to the final 
result. This is essentially the same calculation as was performed previously in [14] without the zero mode. We emphasize again that our formalism allows a quick generalization to the case of non-vanishing zero-mode.

We see from (3.13) that the set of de Sitter-invariant states $\left\{\left|\Psi_{i}\right\rangle\right\}$ built from the seed states $\left.\left\{\llbracket \psi_{i}\right\rangle\right\}$ forms an orthonormal set. One can also show that this set spans the space of de Sitter-invariant states constructed from linear combinations of auxiliary states with $E>1$. The proof is exactly as in [14]. Thus we have an orthonormal basis of states as desired.

We conclude this section with a discussion of auxiliary states with $E \leq 1$. It is natural to ask whether such states contribute to the physical Hilbert space and, if so, how we can incorporate them into our formalism. Fortunately, for any vacuum there are only a few such states (and there are none for $h+\tilde{h}>1$ ). In particular,

The case $p=w=0$ : The only states with $E \leq 1$ are the vacuum $\llbracket 0 ; 0,0\rangle$ and the single-particle states $\left.\left.\alpha_{-1} \rrbracket 0 ; 0,0\right\rangle, \widetilde{\alpha}_{-1} \rrbracket 0 ; 0,0\right\rangle$. The vacuum is de Sitter-invariant and can be a state in the physical Hilbert space. However, this state must be treated separately since for $\llbracket 0 ; 0,0\rangle$ group averaging does not converge. This separate treatment may be justified via the observation (see [18, 33]) that $\llbracket 0 ; 0,0\rangle$ is superselected from states where group averaging does converge. Turning now to the single-particle states, one notes that they each have angular momentum \pm 1 . As a result, the group average of such states over the rotation group $\mathrm{SO}(2) \subset \mathrm{SO}(2,1)$ already vanishes and we do not expect these states to contribute the physical Hilbert space.

The case $p^{2} \leq 1, w=0$, or $p=0, R^{2} w^{2} / 4 \leq 1$ : Here the only states with $E \leq 1$ are vacua $\llbracket 0 ; h, \widetilde{h}\rangle$. Such vacua are not de Sitter invariant, though group averaging again fails to converge. In this case we expect an appropriately renormalized form of group averaging to converge, though we leave this for future work. The resulting de Sitter-invariant states will again be superselected from states for which no such renormalization was needed. See [18, 33, 34, 35, 36] for further examples and discussion of this phenomenon.

The case $|k|=|w|=1$ and $R=\sqrt{2}$ : Such states also have $J= \pm 1 \neq 0$ and again group average to zero under $\mathrm{SO}(2) \subset \mathrm{SO}(2,1)$. We expect no physical states from such seed states.

\section{Physical Entropy}

We now compute the density of physical states. One typically computes this density as a function of energy. However, as previously remarked, there is no natural conserved notion of energy in de Sitter space. Moreover, those charges which are associated with de Sitter isometries must vanish for physical states. We will thus need to find some other notion of energy to use below.

A natural approach is to follow [22] and to consider the energy $E$ defined in section 2. which measures the flux of stress energy through the surface $\tau=0$. Since the definition 
of $E$ as the eigenvalue of $H$ is not de Sitter invariant, this quantity is not a priori defined on physical states. Nevertheless, a de Sitter-invariant notion of $E$ was defined in [22]. In our present language, the de Sitter invariant energy is the operator whose eigenstates are precisely the physical states $\left|\Psi_{i}\right\rangle$ obtained by group averaging Higuchi seed states $\left.\llbracket \psi_{i}\right\rangle_{\text {seed }}$, and such that the eigenvalue of $\left|\Psi_{i}\right\rangle$ is just the eigenvalue of $H$ for $\left.\llbracket \psi_{i}\right\rangle_{\text {seed }}$. The one to one map between physical states and seed states, and that fact that the $\left|\Psi_{i}\right\rangle$ form an orthogonal basis of $\mathcal{H}_{\text {phys }}$, imply that this de Sitter-invariant energy is a self-adjoint operator on $\mathcal{H}_{\text {phys }}$. Furthermore, because any two states related by the action of some $U(g)$ yield the same state under group averaging, we see that defining $H$ in some other reference frame (i.e., replacing $H$ by $U(g) H U\left(g^{-1}\right)$ for some $g$ in the definition of a Higuchi seed state) would lead to the same de Sitter-invariant notion of energy. In a very rough sense, this energy operator considers the energy flux of a physical state through each possible de Sitter neck (associated with each possible choice of reference frame) and reports the smallest value obtained. For simplicity, we will again use $E$ to denote the eigenvalue of this de Sitter-invariant energy.

It is clear that counting the density of physical states is equivalent to counting the number of Higuchi seed states as a function of $E$. The density diverges when the scalar target space is non-compact, so we focus on the case with $S^{1}$ target space. As usual, we perform the calculation separately for the Fock space over each vacuum $\llbracket 0 ; h, \widetilde{h}\rangle$. Our task is thus to compute $\ln \mathcal{N}_{\text {seed }}(N)$, the logarithm of the number of Higuchi seed states as a function of the level $N$ (recall $N=E-h-\widetilde{h}$ ) above each vacuum $\llbracket 0 ; h, \widetilde{h}\rangle$. To do so, we must first examine the seed state criteria in more detail. We begin with $S O(2)$ invariance which requires

$$
J \llbracket n, \widetilde{n} ; h, \widetilde{h}\rangle=(k w+n+\widetilde{n}) \llbracket n, \widetilde{n} ; h, \widetilde{h}\rangle=0,
$$

so that an $S O(2)$-invariant state at level $N$ must satisfy

$$
n=\frac{1}{2}(N-k w), \quad \widetilde{n}=\frac{1}{2}(N+k w) .
$$

Since $n$ and $\tilde{n}$ must be non-negative integers, $S O(2)$-invariant states are possible only at levels $N=k w+2 m$, where $m$ is a non-negative integer.

Next consider the property $\left.L_{1} \rrbracket \psi\right\rangle_{\text {seed }}=0$. Because the left- and right-moving sectors commute, we can decompose the Fock space over a given vacuum into left- and right-moving Hilbert spaces $\mathcal{H}_{\text {aux }}=\mathcal{H}_{\mathrm{L}} \otimes \mathcal{H}_{\mathrm{R}}$; an auxiliary state with levels $(n, \widetilde{n})$ is then in the product space $\mathcal{H}_{n} \otimes \mathcal{H}_{\tilde{n}}$. Because $L_{1}$ acts only on left-movers we can focus on $\mathcal{H}_{n}$. The annihilation operator $L_{1}$ lowers $n$ by 1 , i.e. $\left.\left.L_{1} \rrbracket n, \widetilde{n} ; h, \widetilde{h}\right\rangle=\llbracket n-1, \widetilde{n} ; h, \widetilde{h}\right\rangle$. In fact, $L_{1}$ is a surjective map from $\mathcal{H}_{n}$ to $\mathcal{H}_{n-1}$ :

$$
L_{1}: \mathcal{H}_{n} \rightarrow \mathcal{H}_{n-1} .
$$

The number of states in $\mathcal{H}_{n}$ annihilated by $L_{1}$ is therefore given by the difference in dimension

$$
\left(\begin{array}{c}
\# \text { states in } \mathcal{H}_{n} \\
\text { annihilated by } L_{1}
\end{array}\right)=\operatorname{dim}\left(\mathcal{H}_{n}\right)-\operatorname{dim}\left(\mathcal{H}_{n-1}\right)=P(n)-P(n-1),
$$

where $P(x)$ is the number of integer partitions in $x$ [28]. For $n=1$ we have $P(1)-P(0)=0$, while for $n>1$ we have $P(n)-P(n-1)>0$. The same argument applies for the action 
of $\widetilde{L}_{1}$ on the right-moving sector $\mathcal{H}_{\widetilde{n}}$. Combining our observations (4.2) and (4.4), we find that the number of seed states at level $N$ is

$$
\begin{aligned}
\mathcal{N}_{\text {seed }}(N)= & P\left(\frac{N+k w}{2}\right)\left[P\left(\frac{N-k w}{2}\right)-P\left(\frac{N-k w-2}{2}\right)\right] \\
& \times P\left(\frac{N-k w}{2}\right)\left[P\left(\frac{N+k w}{2}\right)-P\left(\frac{N+k w-2}{2}\right)\right] .
\end{aligned}
$$

One can easily re-write this as a function of the energy flux $E$, though in practice this will not be required to establish agreement with the density of states in $\mathcal{H}_{\text {aux }}$.

Let us now compare the number of seed and auxiliary states for both small and large $N$. Note that the number of states in $\mathcal{H}_{\text {aux }}$ with given $(n, \widetilde{n}, h, \tilde{h})$ is $\operatorname{simply} \mathcal{N}_{\text {aux }}(n, \widetilde{n})=$ $P(n) P(\widetilde{n})$ and the total number of states at level $N$ is

$$
\mathcal{N}_{\text {aux }}(N)=\sum_{n^{\prime}=0}^{N} P\left(n^{\prime}\right) P\left(N-n^{\prime}\right) .
$$

Because $P(x) \sim x$ for $x$ of order 1 , for small $N$ there are dramatically fewer seed states than auxiliary states:

$$
\mathcal{N}_{\text {seed }}(N \sim 1) \sim N, \quad \mathcal{N}_{\text {aux }}(N \sim 1) \sim N^{2}
$$

However, it is more interesting to compare entropies in the thermodynamic limit of large $N$. For $N \rightarrow \infty$ we may use the Hardy-Ramanujan formula for the asymptotic behavior of $P(x)$ 37]:

$$
P(x) \approx \frac{1}{4 x \sqrt{3}} \exp \left[2 \pi \sqrt{\frac{x}{6}}\right] \text { as } x \rightarrow \infty .
$$

Inserting (4.8) into (4.5) and using Cardy's formula 38 to compute (4.6) yields

$$
S_{\text {seed }}(N) \approx S_{\text {aux }}(N) \approx \frac{2 \pi}{\sqrt{3}} \sqrt{N}
$$

so that in this limit the seed state entropy agrees with the entropy of the auxiliary Hilbert space as claimed. This provides an explicit confirmation of the general argument given in the appendix of [22].

\section{Discussion}

We have studied the behavior of $1+1$ massless scalars under de Sitter group averaging, building on earlier work by Higuchi [14]. The new element was to include the scalar zero mode. We constructed an orthonormal set of de Sitter-invariant states which forms a basis of the physical state space (up to the minor exceptions discussed in section 3). We have also computed the entropy of this physical space. As anticipated in [22], to leading order at large $E$ this entropy agrees with the entropy of the auxiliary Hilbert space. This observation supports the claim that group averaging will yield enough states to reproduce classical physics in the $\hbar \rightarrow 0$ limit. 
The elegant orthonormal bases constructed constructed here and in [14] belie the fact that implementing group averaging for more general fields can be quite difficult (see e.g. 223]). The simplicity of group averaging for the present model is in part due working in low dimensions, but has more to do with the presence of conformal symmetry. For general masses and dimensions one looses the useful commutation relations between the raising and lowering parts of $B_{1}$ (here $L_{1}+\tilde{L}_{1}$ and $L_{-1}+\tilde{L}_{-1}$ ) and $E$ which in our case followed from the Virasoro algebra. Similar relations do hold, however, for conformally-coupled scalar fields in arbitrary dimension, i.e. for scalars on $d S_{d+1}$ which satisfy the equation of motion

$$
g^{a b} \nabla_{a} \nabla_{b} \phi(x)=\left(\frac{d^{2}-1}{4}\right) \phi(x) .
$$

In such cases it is straightforward to use Higuchi's algorithm to construct an orthonormal basis of physical states. (The $3+1$ case was studied in [39].) Additionally, in the right choice of gauge, both free gravitons and free gauge vector fields in $3+1$ dimensions have boost matrix elements identical to those of conformally coupled scalar fields, and one may again construct an orthonormal basis [14.

The more general case remains open for future work. However, one expects the massless scalar field in higher dimensions to be qualitatively similar to the case discussed here. I.e., we expect that there is some analogue of our quantity $E$ which in some sense measures the total excitation of the state, including contributions from both particles and the zero mode. One expects group averaging to fail when the quantity is very small, but to converge when it is sufficiently large.

\section{Acknowledgements}

The authors are grateful to Atsuchi Higuchi for many discussions of group averaging and for sharing his unpublished notes [39]. This work was supported in part by the US National Science Foundation under Grant No. PHY05-55669, and by funds from the University of California.

\section{References}

[1] E. Komatsu et al. [WMAP Collaboration], "Five-Year Wilkinson Microwave Anisotropy Probe (WMAP) Observations:Cosmological Interpretation," arXiv:0803.0547 [astro-ph].

[2] A. G. Riess et al. [Supernova Search Team Collaboration], "Observational Evidence from Supernovae for an Accelerating Universe and a Cosmological Constant," Astron. J. 116, 1009 (1998) [arXiv:astro-ph/9805201].

[3] M. Rees, Before the beginning: our universe and others (Simon and Schuster, New York, 1997), p 221.

[4] T. Banks, "Cosmological breaking of supersymmetry or little Lambda goes back to the future. II," arXiv:hep-th/0007146.

[5] E. Witten, "Quantum gravity in de Sitter space," arXiv:hep-th/0106109.

[6] D. Brill \& S. Deser, "Instability of closed spaces in general relativity," Commun. Math. Phys. 32291 (1973). 
[7] V. Moncrief, "Space-Time Symmetries And Linearization Stability Of The Einstein Equations. 1," J. Math. Phys. 16, 493 (1975).

[8] V. Moncrief, "Space-Time Symmetries And Linearization Stability Of The Einstein Equations. 2," J. Math. Phys. 17, 1893 (1976).

[9] V. Moncrief, "Invariant States And Quantized Gravitational Perturbations," Phys. Rev. D 18, 983 (1978).

[10] J. Arms, "Linearization stability of the Einstein-Maxwell system," J. Math. Phys. 18, 830 (1977); "On coupled gravitational and gauge fields" J. Math. Phys. 20, 443 (1979).

[11] A. E. Fisher and J. E. Marsden, in General Relativity: An Einstein Centenary Survey, edited by S. W. Hawking and W. Israel (Cambridge University Press, Cambridge, England, 1979).

[12] J. M. Arms, J. E. Marsden and V. Moncrief, "The Structure Of The Space Of Solutions Of Einstein's Equations. Ii. Several Killing Fields And The Einstein Yang-Mills Equations," Annals Phys. 144, 81 (1982).

[13] A. Higuchi, "Quantum linearization instabilities of de Sitter space-time. 1," Class. Quant. Grav. 8, 1961 (1991).

[14] A. Higuchi, "Quantum linearization instabilities of de Sitter space-time. 2," Class. Quant. Grav. 8, 1983 (1991).

[15] B. Losic and W. G. Unruh, "On leading order gravitational backreactions in de Sitter spacetime," Phys. Rev. D 74, 023511 (2006)

[16] N. P. Landsman, "Rieffel induction as generalized quantum Marsden-Weinstein reduction," arXiv:hep-th/9305088.

[17] D. Marolf, Ph.D Thesis (1992); "Quantum observables and recollapsing dynamics," Class. Quant. Grav. 12, 1199 (1995) [arXiv:gr-qc/9404053].

[18] A. Ashtekar, J. Lewandowski, D. Marolf, J. Mourao and T. Thiemann, "Quantization of diffeomorphism invariant theories of connections with local degrees of freedom," J. Math. Phys. 36, 6456 (1995) [arXiv:gr-qc/9504018].

[19] D. Giulini and D. Marolf, "A uniqueness theorem for constraint quantization," Class. Quant. Grav. 16, 2489 (1999) [arXiv:gr-qc/9902045].

[20] D. Marolf, "Group averaging and refined algebraic quantization: Where are we now?," arXiv:gr-qc/0011112.

[21] D. Giulini and D. Marolf, "On the generality of refined algebraic quantization," Class. Quant. Grav. 16, 2479 (1999) [arXiv:gr-qc/9812024].

[22] S. B. Giddings and D. Marolf, "A global picture of quantum de Sitter space," Phys. Rev. D 76, 064023 (2007) [arXiv:0705.1178 [hep-th]].

[23] D. Marolf and I. A. Morrison, "Group Averaging for de Sitter free fields," in preparation.

[24] B. Allen and A. Folacci, "The massless minimally coupled scalar field in de Sitter space," Phys. Rev. D 35, 3771 (1987).

[25] E. Mottola, "Particle Creation In De Sitter Space," Phys. Rev. D 31, 754 (1985).

[26] D. Polarski, "Massive scalar field on the static de Sitter space," Phys. Rev. D 41, 2519 (1990). 
[27] P. Di Francesco, P. Mathieu and D. Senechal, "Conformal Field Theory," New York, USA: Springer (1997) $890 p$

[28] P. H. Ginsparg, "Applied Conformal Field Theory," arXiv:hep-th/9108028.

[29] E. Joung, J. Mourad and R. Parentani, "Group theoretical approach to quantum fields in de Sitter space II. The complementary and discrete series," JHEP 0709, 030 (2007) [arXiv:0707.2907 [hep-th]].

[30] B. Allen, "Vacuum States In De Sitter Space," Phys. Rev. D 32, 3136 (1985).

[31] V. Bargmann, "Irreducible unitary representations of the Lorentz group," Ann. of Math. (2) 48, $568(1947)$

[32] N. Ya. Vilenken, and A. U. Klimyk, "Representations of Lie Groups and Special Functions," vols 1-3. (Dordrecht: Kluwer Acad. Publ. 1991-1993).

[33] D. Marolf, "Refined algebraic quantization: Systems with a single constraint," arXiv:gr-qc/9508015

[34] A. Gomberoff and D. Marolf, "On group averaging for SO(n,1)," Int. J. Mod. Phys. D 8, 519 (1999) [arXiv:gr-qc/9902069].

[35] J. Louko, "Group averaging, positive definiteness and superselection sectors," J. Phys. Conf. Ser. 33, 142 (2006) [arXiv:gr-qc/0512076].

[36] J. Louko and A. Molgado, "Superselection sectors in the Ashtekar-Horowitz-Boulware model," Class. Quant. Grav. 22, 4007 (2005) [arXiv:gr-qc/0505097].

[37] G. H. Hardy, "An introduction to the theory of numbers," Oxford 1962, 421p

[38] J. L. Cardy, "Operator Content Of Two-Dimensional Conformally Invariant Theories," Nucl. Phys. B 270, 186 (1986).

[39] A. Higuchi, unpublished notes. 